\title{
Waveguide structures for efficient evanescent field coupling to zero mode waveguides
}

\section{Sarkar}

\section{A. J. H. Wachters}

\section{H. P. Urbach}

\section{J. J. H. B. Schleipen jean.schleipen@philips.com}

\section{P. J. van der Zaag}

\section{R. Wimberger-Friedl} Optics Research Group, Delft University of Technology, Lorentzweg 1, 2628 CJ Delft, The Netherlands
Institut d'Optique Graduate School, 2 Avenue Augustin Fresnel, 91127 Palaiseau, France

Optics Research Group, Delft University of Technology, Lorentzweg 1, 2628 CJ Delft, The Netherlands

Optics Research Group, Delft University of Technology, Lorentzweg 1. 2628 CJ Delft, The Netherlands

Philips Research Laboratories, High Tech Campus 34, 5656 AE Eindhoven, The Netherlands

Philips Research Laboratories, High Tech Campus 11, 5656 AE Eindhoven, The Netherlands

Philips Research Laboratories, High Tech Campus 11, 5656 AE Eindhoven, The Netherlands

The use of waveguide structures is examined to improve the efficiency of evanescent field coupling into zero-mode waveguides. Model calculations show that waveguide excitation using diffractive structures, increases the magnitude of the evanescent electric field by an order of magnitude compared to far field excitation of the evanescent field. A more efficient excitation of fluorescent markers used in e.g. sequencing instrumentation ultimately enables real-time single molecule detection using laser systems with moderate output power. [DOI: http://dx.doi.org/10.2971/jeos.2014.14019]

Keywords: Zero mode waveguide, DNA sequencing, waveguide structure, grating structure, finite element methods

\section{INTRODUCTION}

Ever since the introduction of so-called next generation sequencing in 2005 [1], the speed at which DNA, or more specifically nucleic acids, can be sequenced has increased rapidly. The increase of sequencing performance in next generation sequencing is enabled by the massive parallelization of the detection of the sequencing reaction, such that multiple parts on a nucleic acid are detected at the same time. Predominantly, the systems currently available for sequencing rely on clonal amplification of the DNA followed by optical detection [1]-[3]. The clonal amplification ensures that the optical signal upon incorporation of a labeled fluorophore is amplified, thereby improving the signal to noise ratio. However, the bias introduced by the clonal amplification, which typically relies on some form of amplification by polymerase chain reaction (PCR), subsequently led to the development of systems which do direct single molecule detection, i.e. without a PCR-based amplification step $[4,5]$. Of these, the system of Pacific Biosciences is of particular interest as it relies on single molecule real-time (SMRT) sequencing, enabled by the use of a nanophotonic, so-called zero-mode waveguide (ZMW) [6], in which the incorporation of labeled nucleotides by a polymerase molecule bound to the $\mathrm{ZMW}$ is followed in real-time. A dedicated holographic phase mask generates a 2-dimensional array of thousands of focused light beams, each illuminating a ZMW structure and exciting an evanescent field for probing the sequencing reaction [7]. The advantage of this approach for sequencing is that 'reads' of the DNA fragments are generated at relatively high speed compared to other optical methods, because the need for advanced chemical processing required to start nucleotide incorporation reaction is eliminated. Consequently, the rate at which a nucleotide will be incorporated in the sequencing reaction is no longer determined by microfluidic wash steps, but merely by the rate at which a polymerase is incorporating the bases. Typically this could be in the base/ms range. However, in the current SMRT sequencing system this has been slowed down to 1-3 base/s [5]. Still, this renders the sequencing speed of SMRT sequencing two orders of magnitude faster than approaches based on clonal amplification and optical detection [1]-[3]. Additionally, by monitoring the time it takes for the incorporation of a nucleotide, this system can discern the incorporation of methylated bases [8], which is important from biological perspective, as methylation of DNA plays an important role in gene regulation and thereby cancer.

Optically the disadvantage of this system is that all the zeromode waveguides (each holding a DNA fragment to be sequenced) have to be excited in parallel [7] and need to be monitored continuously, as the incorporation of nucleotides is a stochastic process. This requires rather high laser powers as each ZMW needs to be driven preferably close to saturation. From elementary consideration, using typical numbers for the absorption cross section and excited state lifetime of dyes, the saturation power $P$ per $\mathrm{ZMW}$ is typically a few $\mathrm{mW} / \mu \mathrm{m}^{2}$. 
Since the overall laser power scales linearly with the number of ZMWs, this leads to a required excitation power of around $10 \mathrm{~W}$ when several thousands of ZMWs are to be used. In view of the need to come to more efficient and integrated cartridgebased systems $[9,10]$ for routine clinical adoption of DNA sequencing, the use of waveguide structures has been examined. This with the aim to exploit the fact that light inside the evanescent field inside the ZMW is not propagating and can be 'reused' for excitation of other ZMWs by using a waveguide, thereby reducing the required laser power needed for exciting these ZMW arrays.

The paper is organized as follows. After a description of the used optical modeling techniques, two different approaches will be discussed, using diffractive optical structures in order to achieve a more efficient ZMW evanescent field excitation: one in which the excitation is perpendicular and an alternative in which the excitation is along the plane of the ZMWs. The outcome of both approaches will be discussed in terms of feasibility and effectiveness when being applied in an experimental setting.

\section{MODELING}

Wiregrids consist of periodically spaced, thin metallic strips on a transparent dielectric substrate, and are readily being used e.g. as element in display applications [11]. These wiregrids can be considered as one-dimensional ZMW waveguides and an example of such a wiregrid is given in Figure 1(a). The TE electric field is not allowed to propagate in such waveguides whenever the gap between the two metal wires is smaller than half the wavelength of the light. Instead an evanescent field will be excited in the waveguide which is localized within distances less than a wavelength perpendicular to the wiregrid substrate. These well localized fields in between the metal wires may excite single molecules for e.g. SMRT sequencing. Two methods will be described for exciting this evanescent field inside the wiregrid waveguides. The first one relies on focusing an EM field on the ZMW structures using diffractive optical elements. The second method uses a metal-insulator-metal (MIM) waveguide with a TE polarized input field and the ZMWs embedded in one of the metal cladding layers. The schematic of these designs is shown in Figure 1.

\subsection{Binary phase gratings}

We can use a binary phase grating (BPG) by manipulating the diffraction orders in the near field $(\approx 100 \mu \mathrm{m})$ to have a focusing effect of the field where the ZMWs are located. Considering all the orders diffracted from the grating we evaluate the total field in the substrate and then manipulate the grating parameters to have the desired field distribution at the ZMW plane.

The grating with the substrate and the metal ZMW layer is shown in Figure 2. The electric fields reflected and transmitted

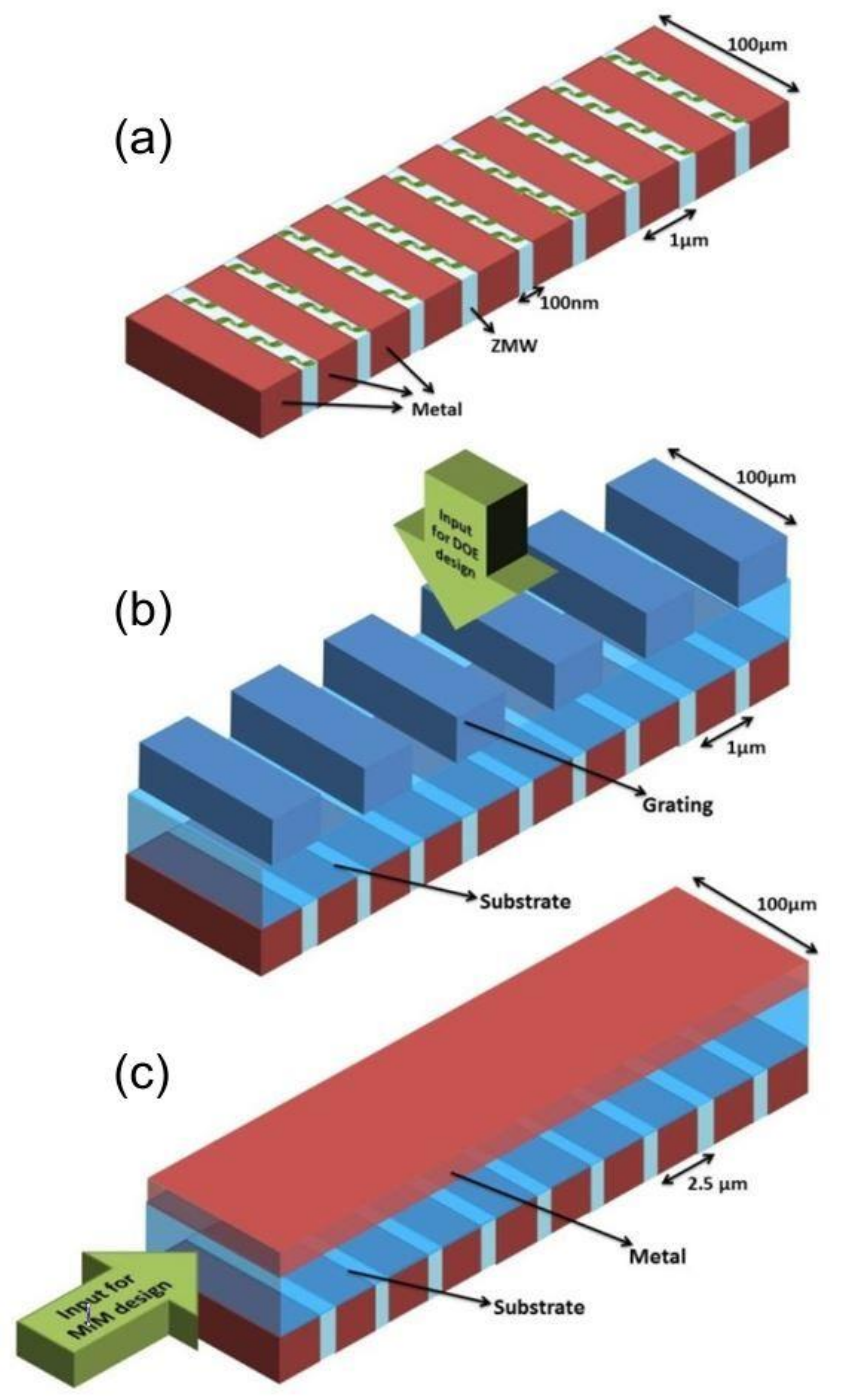

FIC. 1 Schematic of a wire grid and the two methods for exciting an evanescent field. a) The space between two neighboring wires of a wiregrid acts as a ZMW where an evanescent field is created, used for probing molecules in a surface sensitive way. $b$ ) For the grating design we have a plane wave incident from the top of the substrate where a grating is used to increase the field in the ZMWs. c) For the MIM design the light would be incident from the side of the wiregrid substrate using an additional waveguide with a metal cladding layer.

by the grating are given by [12]:

$$
\begin{aligned}
& E_{\text {incident }}=\exp \left[-j k_{0} n_{1}\{\sin (\theta) x+\cos (\theta) z\}\right] \\
& E_{\text {reflected }}=E_{\text {incident }}+\sum_{i} R_{i} \exp \left[-j\left(k_{x i} x+k_{1, z i} z\right)\right] \\
& E_{\text {transmitted }}=\sum_{i} T_{i} \exp \left[-j\left(k_{x i} x+k_{2, z i} z\right)\right]
\end{aligned}
$$

where $i$ is the diffraction order, and $R_{i}$ and $T_{i}$ are the normalized electric field amplitudes for the $i^{\text {th }}$ reflected and transmitted waves respectively. The propagation constants for the fields are given by

$$
\begin{aligned}
& k_{l, z i}=k_{0}\left[n_{l}^{2}-\left(\frac{k_{x i}}{k_{0}}\right)^{2}\right]^{1 / 2} \quad l=1,2 ; i=0,1,2, \ldots \\
& k_{x i}=k_{0}\left[n_{1} \sin (\theta)-\frac{i \lambda_{0}}{\Lambda}\right]
\end{aligned}
$$

We have assumed an implicit time dependence given by $\exp (-j \omega t)$ where $\omega>0$. With the metal ZMW layer below 


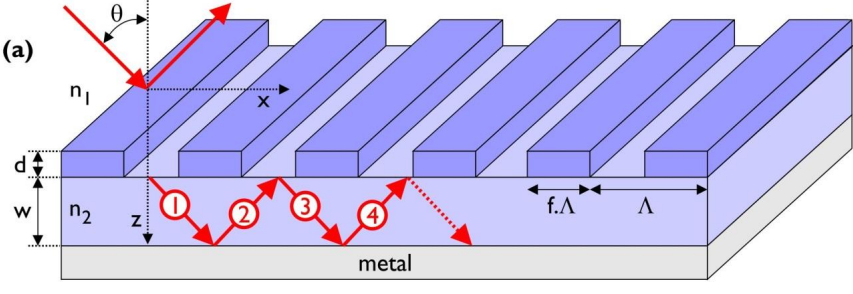

(b)

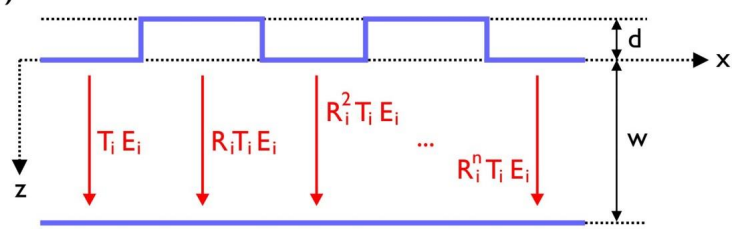

FIG. 2 Diagram of the binary phase grating with the substrate and the metal layer shown in (a). Here $\Lambda$ is the grating period, $f$ the grating fraction and $d$ the grating height. Considering $E_{i}$ to be the input electric field, the fields reflected from the grating in the substrate is shown in (b).

the substrate, the field transmitted by the grating (forward wave 1 in Figure 2) is reflected back into the substrate (backward wave 2 in Figure 2). Now, if $R_{i}$ for this backward wave is much larger than $T_{i}$, the field is again reflected back into the substrate (forward wave 3 in Figure 2) and thus we have a sustained field in the substrate due to multiple reflections from the grating and the metal layer. This is schematically shown in Figure 2(b) where the reflection of the metal ZMW layer is assumed to be $100 \%$. The total electric field is thus an infinite sum of all the reflected components of the field for all grating orders, and is given by the following expression (TE polarized):

$$
\begin{aligned}
& E_{\text {subs }}= \sum_{n} \sum_{i}\left\{R_{i}^{n} T_{i} \exp \left[-j\left(k_{x i} x-k_{2, z i} z\right)\right]\right\} \\
&+\sum_{n} \sum_{i} R_{i}^{n} T_{i} \exp \left[-j\left(k_{x i} x-k_{2, z i}(2 w-z)\right\}\right] \\
&=\sum_{i=1}^{\infty} T_{i} \frac{\exp \left[-j k_{x i} x\right]\left\{\exp \left[j k_{2, z i} z\right]+\exp \left[j k_{2, z i}(2 w-z)\right]\right\}}{1-R_{i}}
\end{aligned}
$$

The performance of these binary phase gratings in terms of increasing the electric field at the ZMW locations will be discussed with some model assumptions based on typical experimental conditions. A wiregrid ZMW substrate has a typical grid periodicity of several microns, hence $\Lambda=1 \mu \mathrm{m}$. The grating fraction $f$, being the ratio of the width of the higher refractive index (ridge) material to that of the lower refractive index material (groove), is chosen to be 0.7. With the ZMWs aligned at the center of the grating ridges (higher refractive index) a larger ridge width maximizes the field at its center. The substrate is chosen to have the same material as the grating ridge for simplicity and to reduce reflection losses at the grating substrate boundary. The refractive index of the substrate is chosen to be 1.6. We consider normal incidence for simplicity as it assures a symmetric distribution of the field along the $y z$-plane. The wavelength of light is taken as $450 \mathrm{~nm}$.

With these parameters the electric field inside the substrate has been evaluated using Eq. (3) for different values of grating height $d$ and substrate thickness $w$. Appropriate values of $d$ and $w$ have been chosen by maximizing the electric field at the metal-substrate interface, directly below the center of the grating ridge where the ZMWs will be located. From this 2-dimensional parameter space, an optimum working point of $d=500 \mathrm{~nm}$ and $w=33 \mu \mathrm{m}$ has been established, bearing in mind the manufacturing requirements for the substrate. The electric field distribution $\left(E_{y}\right)$ in the substrate using Eq. (3) is shown in Figure 3. For reference, the same model system is also solved using a finite elements method (FEM) [13] and the results are also shown in Figure 3. A close resemblance between both field distributions is observed. Any discrepancy may arise due to the assumption of a perfect conductor for the metal for the analytical calculation while the real refractive index of the metal (Al) was used for the FEM calculations. Also for the analytical calculation, an infinite number of reflections in the substrate were assumed for simplicity while in reality, and as shown by the FEM calculations, this assumption does not hold.

Figure 4 shows the mean electric field and intensity in the substrate just above the ZMWs for an input field of $1 \mathrm{~V} / \mathrm{m}$. According to the more exact FEM calculations an increase of the field at each ZMW can be expected of a factor 5.3. The value of the electric energy density $\left(W=\frac{1}{2} \epsilon E_{y}^{2}\right)$ at the position of the ZMW is $35.95 \mathrm{~J} / \mathrm{m}^{3}$, leading to an increase in excitation intensity at each ZMW of several decades:

$$
\frac{W_{\text {evan }}}{W_{\text {input }}}=\frac{\epsilon E_{\text {evan }}^{2}}{\epsilon_{0} E_{\text {input }}^{2}}=\frac{\epsilon}{\epsilon_{0}} E_{\text {evan }}^{2}=71.91
$$

where $E_{\text {evan }}^{2}\left(28.1 \mathrm{~V}^{2} / \mathrm{m}^{2}\right)$ is the average of the square of the field just above the ZMWs as shown in Figure 4 and $\epsilon=2.56$. The overall efficiency of the design with respect to increasing the evanescent field intensity as a function of excitation input power will be discussed in the following sections.

\subsection{Metal-insulator-metal (MIM) waveguides}

An alternative approach to increase the local field intensity inside the ZMW is to use a metal-insulator-metal (MIM) waveguide to guide the light in a thin layer just above the ZMW wiregrid plane, as shown in Figure 5. Since the core of the waveguide has a much smaller cross sectional area than the area illuminated in the grating design, one would expect to achieve a larger gain in overall excitation efficiency using this MIM waveguide solution.

The TE-polarized field inside the MIM will propagate as a sum of several TE polarized guided modes of which the propagation constants $\beta_{m}$, as given by Eq. (5), are real valued [14]. The part of the total field distribution that propagates in the positive $z$-direction is given by [15]:

$$
\begin{aligned}
& E_{\text {total }}(x, z)=\sum_{m} E_{y, m}(x, z) \\
& E_{y, m}(x, z)=a_{m} u_{m}(x) \exp \left(-j \beta_{m} z\right) \\
& u_{m}(x)= \begin{cases}\sqrt{\frac{2}{w}} \cos \left(\frac{m \pi x}{w}\right), & m=1,3,5, \ldots \\
\sqrt{\frac{2}{w}} \sin \left(\frac{m \pi x}{w}\right), & m=2,4,6, \ldots\end{cases} \\
& a_{m}=\sqrt{2 w} E_{0} \\
& \beta_{m}=\sqrt{\left(k_{0} n\right)^{2}-\frac{m^{2} \pi^{2}}{w^{2}}}
\end{aligned}
$$



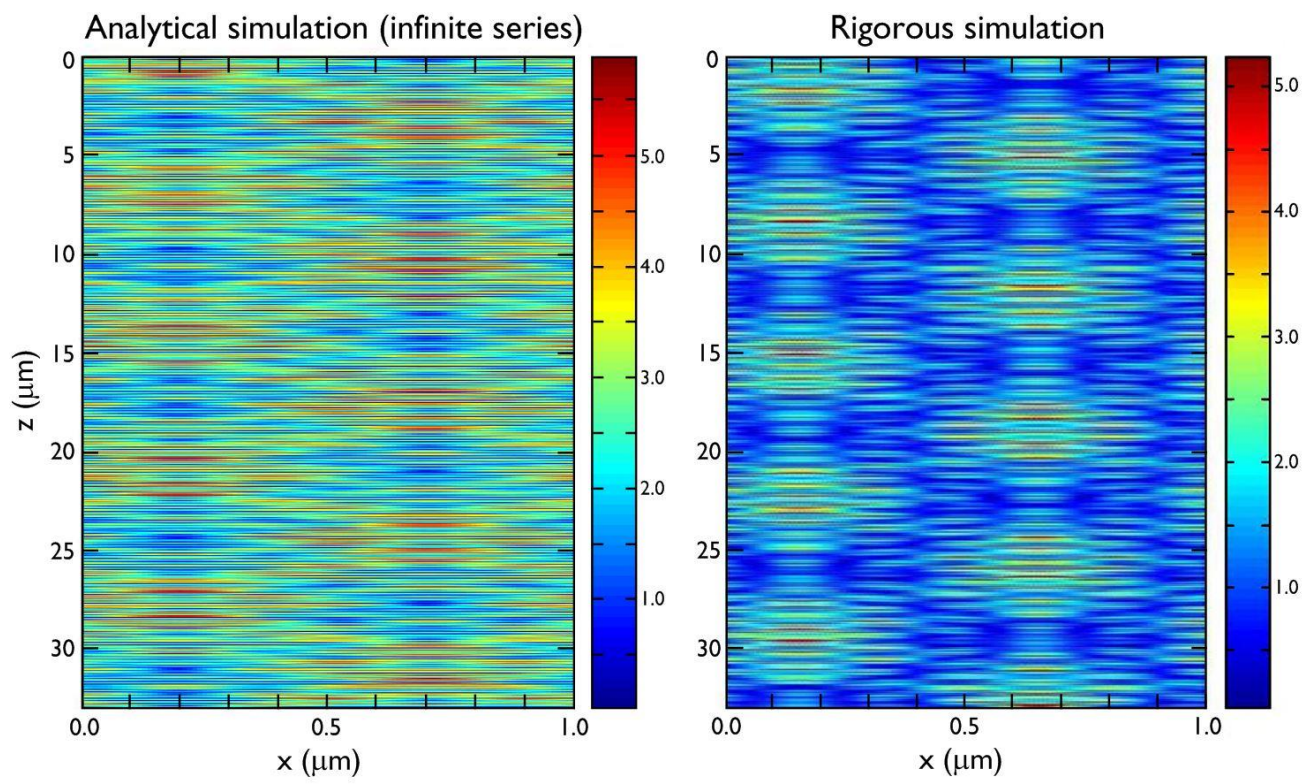

FIG. 3 The field distribution $E_{y}$ is shown in side-view, e.g. as a function of the depth in the substrate $(z)$ for a fixed total substrate thickness $w=33 \mu \mathrm{m}$ and position along the binary phase grating $(x)$. Here $\Lambda=1 \mu \mathrm{m}$, substrate refractive index of $1.6, \lambda=450 \mathrm{~nm}, d=500 \mathrm{~nm}$. The field is calculated analytically using Eq. (3) (left) and also using FEM calculations (right).
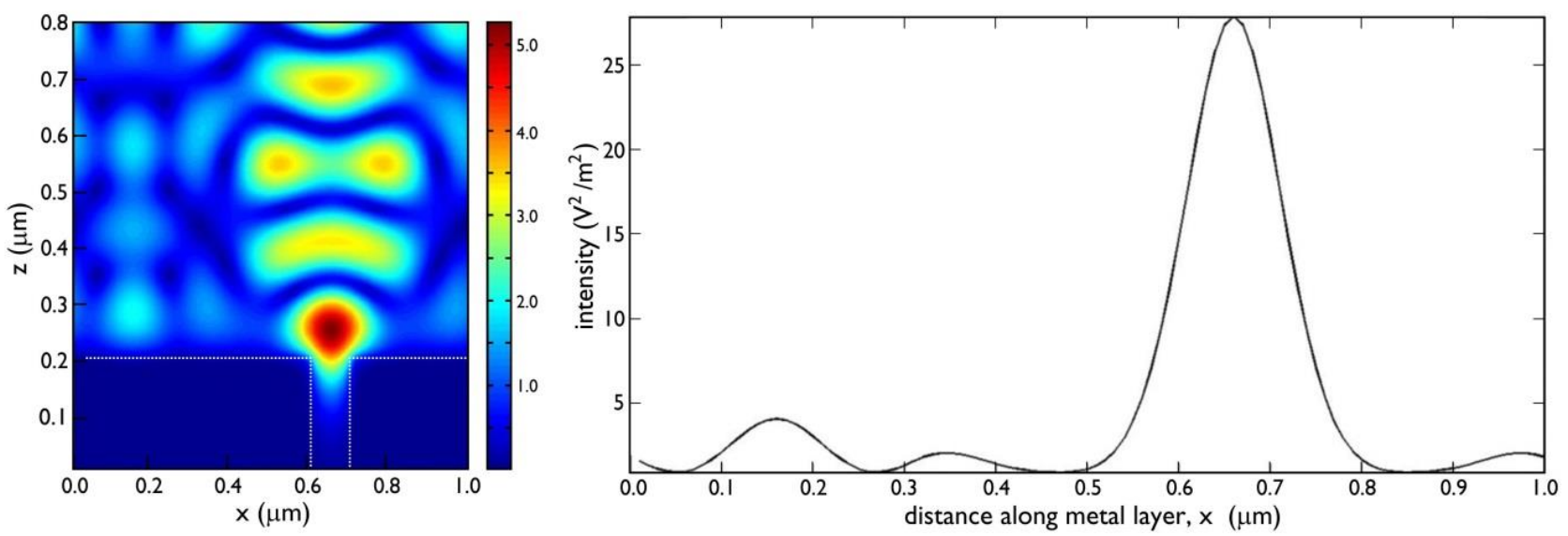

FIG. 4 The field distribution $E_{y}$ near the ZMW for the design shown in Figure 3 (side-view). The field (V/m) is calculated using FEM calculations (left). The intensity distribution $\left(E_{y}^{2}\right)$ at the metal-substrate boundary for $\lambda=450 \mathrm{~nm}$ for the grating design for one ZMW using FEM calculations (right).

Here $k_{0}=2 \pi / \lambda, n$ is the refractive index $(n=1.45)$ and $w$ the height of the waveguide. The metallic walls of the waveguide are assumed to be perfectly conducting.

For maximum coupling of the field into the ZMWs, the field maxima of the propagating modes need to coincide with the actual positions of the zero mode waveguides. Furthermore, ideally only a single mode $(m)$ should propagate inside the waveguide in order to ensure a rather uniform intensity distribution along the propagation direction of the mode. The latter may be achieved by using a binary grating with period $p$ on top of the substrate and matching the propagation constant of one of the grating orders to the propagation constant $\beta_{m}$ of a specific waveguide mode $m$. We should mention here that for the rest of the study the waveguides with only a single propagating mode will be treated, which is assured by using the grating and normal incidence to couple a particular mode in the waveguide.

Due to the finite length $L$ of the waveguide the propagat- ing mode $m$ will be reflected back and forth leading to a standing wave pattern inside the waveguide with periodicity $\pi / \beta_{m}$. The exact locations of the corresponding intensity maxima thereby strongly depend on the waveguide dimensions $L$ and $w$. As a result the local intensity at the position of the ZMWs depends strongly on manufacturing tolerances of the waveguide, which is not acceptable. This can be solved by incorporating an intermediate "bridge" waveguide, having a width $(a)$ of several standing wave periodicities and effectively transferring the core mode intensity towards the ZMWs. This is illustrated in Figure 6 where a bridge waveguide of (e.g.) $400 \mathrm{~nm}$ effectively couples the TE mode electric field to the ZMW regardless of the exact value of $L$.

A drawback of using these relatively wide bridge structures is backscattering of the central mode intensity in the direction opposite to the propagation direction, thereby reducing the overall distance the field can propagate inside the central waveguide. Furthermore the intensity of the propagating mode will gradually decrease due to intrinsic absorption 


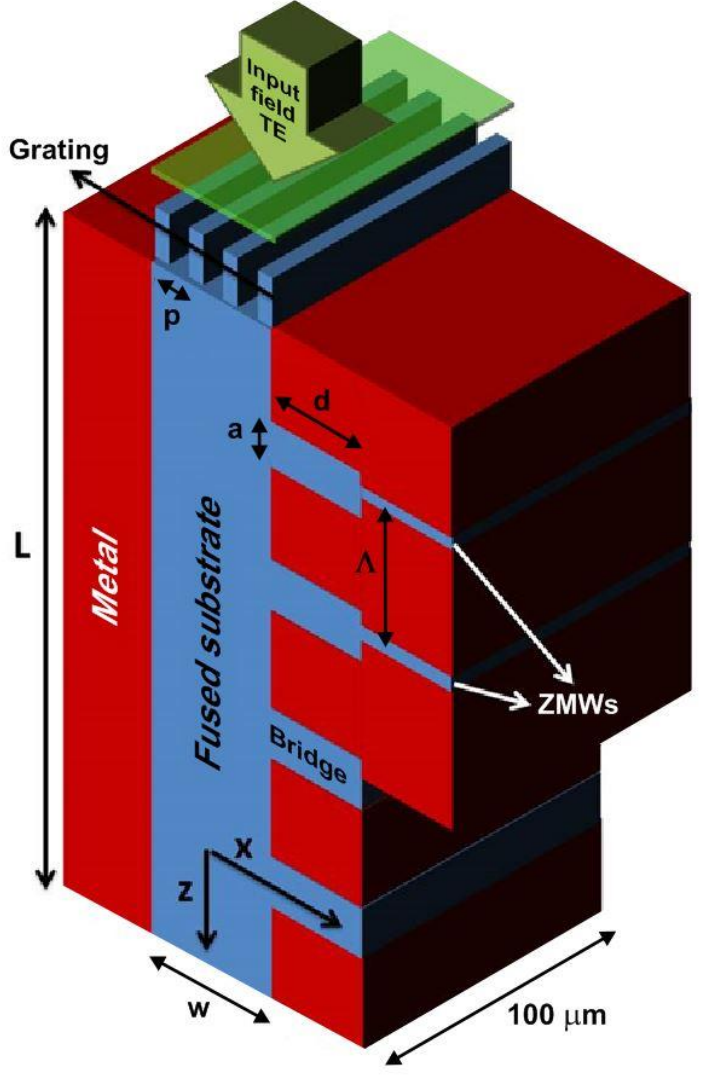

FIC. 5 Schematic of the MIM waveguide design. The grating is used to couple a field with a single $\beta$ in the waveguide so that we have single mode propagation in the waveguide. This helps to have an uniform field in the ZMWs. The 'bridge' structure is needed to reduce the tolerance on the length of the grating.

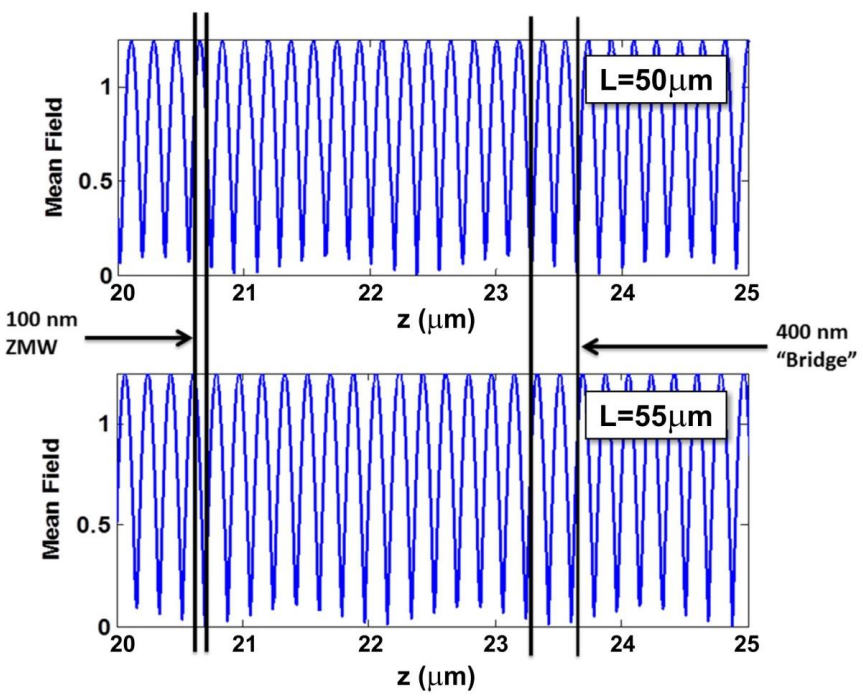

FIG. 6 The mean of the magnitude of the electric field $(m=3)$ at a section of the waveguide is plotted with the distance along the $z$-axis for two values of $L(50 \mu \mathrm{m}$ and $55 \mu \mathrm{m}$ ). With a ZMW of $100 \mathrm{~nm}$ wide (position shown by vertical lines), the coupled field will strongly vary with $L$. However, using a bridge structure with a width of around $400 \mathrm{~nm}$ the total coupled field becomes largely invariant to changing $L$.

at the metal walls of the MIM waveguide. In order to optimize the bridge-waveguide structure for maximum propagation distance we use coupled mode theory [16] to calculate the amount of backscattered light. Here the MIM waveguide is considered as the unperturbed system and the bridge to be a dielectric perturbation. Furthermore we assume that the for- ward wave consists of only one mode that does not change during propagation. The electric field of the back scattered wave can then be deduced as (Appendix A):

$$
\begin{aligned}
A_{p}^{-}= & \text {Constant } \times \sin \left(\beta_{m} a\right) \\
& \times\left\{\frac{\sin \left(\frac{c_{1 p} w}{2}\right)}{c_{1 p}}+\frac{\sin \left(\frac{c_{2 p} w}{2}\right)}{c_{2 p}}\right\} ; p=1,3,5 \ldots \\
C_{1 p}= & \frac{p \pi}{w+d}-\frac{m \pi}{w} \\
C_{2 p}= & \frac{p \pi}{w+d}+\frac{m \pi}{w}
\end{aligned}
$$

where $m$ is the mode in the unperturbed waveguide, and $p$ are all the possible propagating modes present in a waveguide with height $w+d$. The second part of Eq. (6) represents the solution of the overlap integral between the mode of the unperturbed waveguide of height $w$ and the modes of the perturbed waveguide of height $w+d$. The bridge parameters $d$ and $a$ are shown in Figure 5.

The bridge-waveguide design has been simulated thoroughly for different parameters $w, a, d$ and $L$, using the analytic approach of Eq. (6) and FEM calculations. For a large number of bridges $(>10)$ the simulation results start to deviate, due to the failure of the assumptions made in the analytic model. Figure 7 shows the results of FEM calculations on the TE field inside the central waveguide, for an optimum parameter set $d=a=545 \mathrm{~nm}, w=900 \mathrm{~nm}$ and $L=70 \mu \mathrm{m}$, a $3^{\text {rd }}$ order propagating mode being excited inside the waveguide, and with 20 bridges/ZMWs facing the central waveguide. Here the bridges are $2.5 \mu \mathrm{m}$ separated apart and the ZMWs have a width of $100 \mathrm{~nm}$. The input field is TE polarized with an amplitude of $1 \mathrm{~V} / \mathrm{m}$ and the metal is chosen to be aluminum.

Due to scattering and absorption the field intensity gradually decreases along the length of the waveguide. Figure 8 shows the mean intensity at the interface between the bridges and the actual ZMWs. From this the ZMW coupling efficiency with respect to the incoming field can be calculated:

$$
\frac{W_{\text {evan }}}{W_{\text {input }}}=\frac{\epsilon E_{\text {evan }}^{2}}{\epsilon_{0} E_{\text {input }}^{2}}=\frac{\epsilon}{\epsilon_{0}} E_{\text {evan }}^{2}=1.68
$$

where $E_{\text {evan }}^{2}=0.8$ is the average of the square of the fields at the boundary of the ZMW and the bridge as shown in Figure 8 and $\epsilon=2.10$. This intensity gain of 1.68 for the MIM waveguide design is substantially lower as compared to the grating design. However, from an experimental point of view, the ratio between the energy density in the ZMW and the overall total optical power, needed to excite a certain number of ZMWs, is the parameter to be considered. This will be discussed in the following sections.

\subsubsection{MIM waveguide absorption loss}

So far for the analytical calculations we assumed the metal to be a perfect conductor. Taking absorption into account the propagation constant $\beta$ is no longer purely real but has an imaginary part and can be written as $\beta=\beta^{\prime}-i \beta^{\prime \prime}$. The imaginary part $\beta^{\prime \prime}$ is responsible for the attenuation along the length of the waveguide $(z)$ and as a result the field shows an exponential decay expressed as $\exp \left(-\beta^{\prime \prime} z\right)$. For a symmetrical 


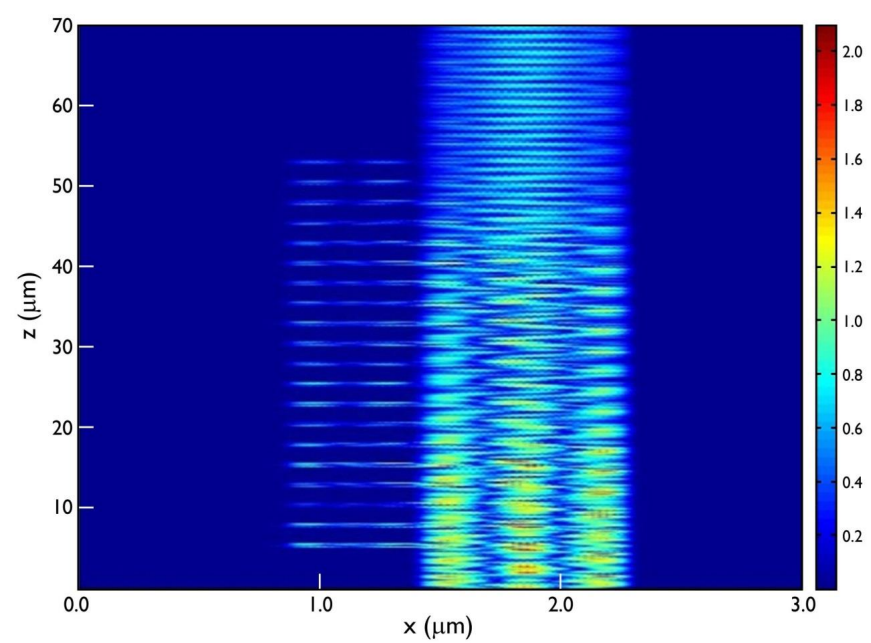

FIG. 7 The magnitude of the TE field (in $\mathrm{V} / \mathrm{m}$ ) in a waveguide with $w=900 \mathrm{~nm}$. The dimension of the bridge is a $\times d=545 \mathrm{~nm} \times 545 \mathrm{~nm}$. Length of the waveguide is $70 \mu \mathrm{m}$ and the separation of the ZMWs is $2.5 \mu \mathrm{m}$. The calculation is done using FEM at $\lambda=450 \mathrm{~nm}$.

\begin{tabular}{|c|c|c|}
\hline $\begin{array}{c}\text { Waveguide } \\
\text { height } \mathbf{w} \\
(\mathbf{n m})\end{array}$ & $\begin{array}{c}\text { Maximum } \\
\text { usable length } \\
(\boldsymbol{\mu m})\end{array}$ & $\begin{array}{c}\text { Max. number } \\
\text { of ZMWs } \\
\left(\boldsymbol{N}_{\text {ZMW }}\right)\end{array}$ \\
\hline 900 & 62.19 & 24 \\
1500 & 103.65 & 41 \\
2100 & 145.11 & 58 \\
2700 & 186.57 & 74 \\
3300 & 228.03 & 91 \\
3900 & 269.49 & 107 \\
4500 & 310.95 & 124 \\
\hline
\end{tabular}

TABLE 1 The total number of available ZMWs as a function of waveguide height $w$. For each height $w$, a single mode is excited with mode number $m=w /(p / 2)$ with $p$ being the period of the grating used to couple the mode into the waveguide. The core refractive index of the waveguide is 1.45 , the metal is aluminum and the wavelength is $450 \mathrm{~nm}$.

MIM waveguide the real and the imaginary parts of the propagation constant can be approximated as (TE modes) [15]:

$$
\begin{aligned}
\beta^{\prime} & =k_{0} \epsilon_{d}^{\frac{1}{2}} \\
\cdot & \left\{1-\frac{1}{2 \epsilon_{d}}\left[\frac{m \pi}{k_{0} w}\right]^{2}\left[1-\left(\frac{2}{k_{0} w}\right) \operatorname{Re}\left\{2\left(\epsilon_{d}-\epsilon_{m}\right)^{\frac{1}{2}}\right\}\right]\right\} \\
\beta^{\prime \prime} & =-k_{0} \epsilon_{d}^{-\frac{1}{2}}\left[m^{2} \pi^{2}\left(k_{0} w\right)^{-3}\right] \operatorname{Im}\left\{2\left(\epsilon_{d}-\epsilon_{m}\right)^{-\frac{1}{2}}\right\}
\end{aligned}
$$

where $k_{0}=2 \pi / \lambda, \epsilon_{d}$ and $\epsilon_{m}$ are the complex dielectric constants of the dielectric core and the metal cladding respectively, $w$ is the central core thickness and $m$ is the order of the mode that propagates in the waveguide.

FEM calculations were done for MIM waveguides of three different heights $(w=900 \mathrm{~nm}, 1500 \mathrm{~nm}, 2100 \mathrm{~nm})$, each with a total length of $70 \mu \mathrm{m}$ and bridge spacing of $2.5 \mu \mathrm{m}$. A grating with period $600 \mathrm{~nm}$ was used to couple a single mode (with $m=3,5,7$ respectively) into the MIM waveguide as described before. The heights of the waveguide have been chosen such that the propagation constant $\beta$ of the guided mode is the same in all three cases, just like the dimension of the bridge for minimum backscattering, as discussed be- fore ( $d=a=545 \mathrm{~nm}$ ). Figure 9(a) shows the mean field along the length of the waveguide $(z)$ as calculated by the FEM for the three different waveguide widths. As expected, the attenuation becomes less for increasing waveguide widths and it follows closely the trend predicted by the analytical formulae of Eq. (8). In Figure 9(b) the intensity of the electric field distribution inside the MIM waveguide is plotted. For all heights $w$ the value of the energy density of the evanescent field at the interface of the bridges and the ZMWs is approximately $1 / 2 \epsilon E_{\text {evan }}^{2}=0.8$. The number of bridges, and corresponding ZMWs, that can be effectively used is governed by the absorption of light along the length of the waveguide. Considering only absorption in the metal, the field decays along the length of the waveguide with an exponential factor $\exp \left(-\beta^{\prime \prime} z\right)$. If we assume that the maximum allowable decrease in field is a factor of 2 , to be able to efficiently couple into the ZMWs, then the total number of ZMWs is given by $N_{\mathrm{ZMW}}=\log (2) /\left(\beta^{\prime \prime} \Lambda\right)$, where the absorption constant $\beta^{\prime \prime}$ depends on the waveguide height $w$, as expressed by Eq. (8) and $\Lambda=2.5 \mu \mathrm{m}$ is the spacing of the bridges. As a result in Table 1, the number of available ZMWs is given as a function of the waveguide width $w$, taking the metal absorption into account.

\section{ZMW EXCITATION EFFICIENCIES}

A proper figure of merit when comparing the binary phase grating and the MIM waveguide designs with plane bulk illumination of $N_{\mathrm{ZMW}}$ zero mode waveguides presently used in SMRT sequencing [7] is the ratio between the energy density of the evanescent electric field inside the ZMWs to the required overall input power of the excitation beam. For a straightforward comparison let us assume that the ZMWs have a footprint $A_{\mathrm{ZMW}}$ of $0.1 \times 100 \mu \mathrm{m}^{2}$ and that they are separated $\Lambda=1 \mu \mathrm{m}$ apart. The efficiency of both designs can now be defined as the input power required to maintain a unit energy density $\left(W_{\text {evan }}\right)$ in the ZMWs:

$$
\begin{gathered}
\eta_{\mathrm{BPG}} \equiv \frac{W_{\text {evan }}}{P_{\text {input }}}=\left(\frac{W_{\text {evan }}}{W_{\text {input }}}\right)_{\text {BPG }} \cdot \frac{1}{c \cdot A_{\text {input }}} \\
=71.91 \cdot \frac{1}{c \cdot N_{\mathrm{ZMW}} \cdot 1 \cdot 100 \mu \mathrm{m}^{2}}=\frac{2.39 \cdot 10^{3}}{N_{\mathrm{ZMW}}}\left[\frac{\mathrm{J} / \mathrm{m}^{3}}{\mathrm{~W}}\right] \\
\eta_{\mathrm{MIM}} \equiv \frac{W_{\text {evan }}}{P_{\text {input }}}=\left(\frac{W_{\text {evan }}}{W_{\text {input }}}\right)_{\mathrm{MIM}} \cdot \frac{1}{c \cdot A_{\text {input }}} \\
=1.68 \cdot \frac{1}{c \cdot w \cdot 100 \mu \mathrm{m}}=\frac{56}{w(\text { in } \mu \mathrm{m})}\left[\frac{\mathrm{J} / \mathrm{m}^{3}}{\mathrm{~W}}\right]
\end{gathered}
$$

where $w$ is the height of the MIM waveguide channel in $\mu \mathrm{m}$ and is related to the number of ZMWs according the results expressed in Table 1.

In order to make a fair comparison of BPG and MIM excitation with plain far field illumination of a series of ZMWs, the evanescent field inside the ZMWs is estimated for far field excitation by calculating the overlap of a diffraction limited Airy illumination profile and the ZMW geometry. For a rectangular shaped ZMW with dimensions $0.1 \times 100 \mu \mathrm{m}^{2}$ and a typical focusing $N A$ of 0.8 this overlap is $\eta_{\text {Airy }}=P_{\mathrm{ZMW}} / P_{\text {Airy }}=0.17$. 


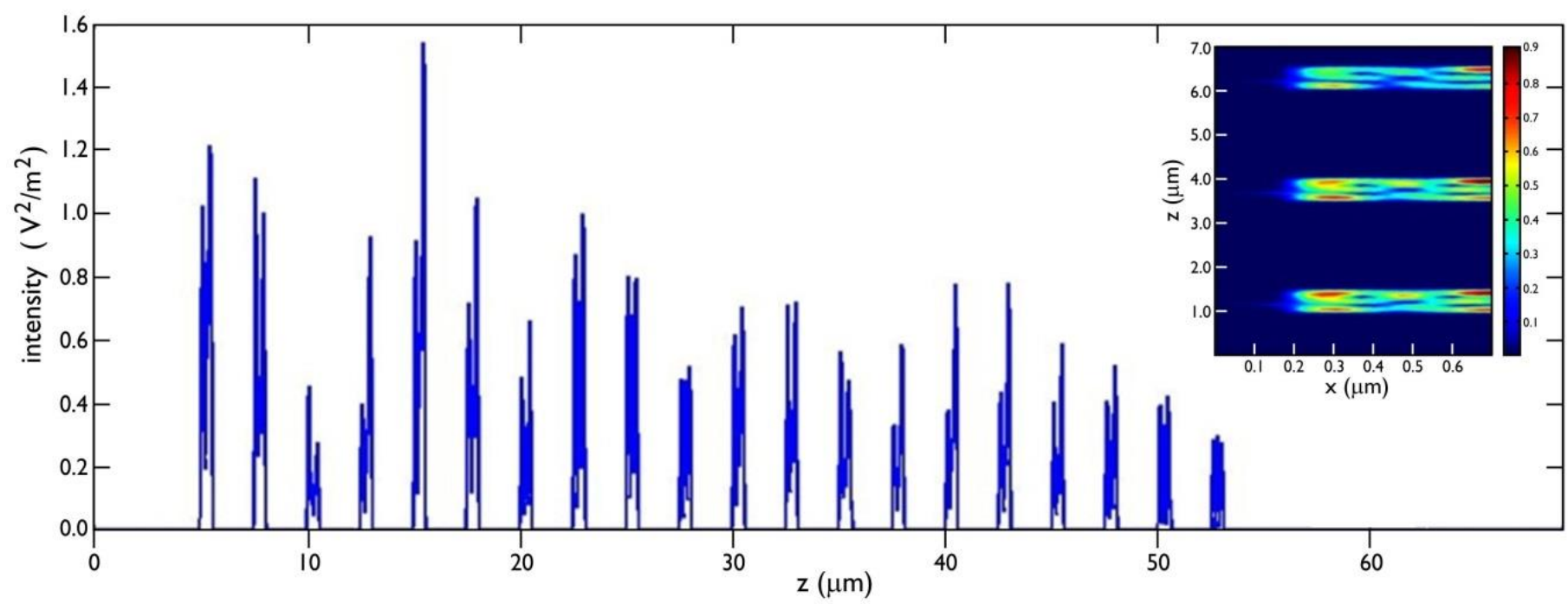

FIG. 8 The intensity distribution at the boundary of the ZMW and the bridges of Figure 7 . The inset shows the field distribution at the $11^{\text {th }}$ to $13^{\text {th }}$ ZMW starting from the input side. The field is calculated using FEM calculations.
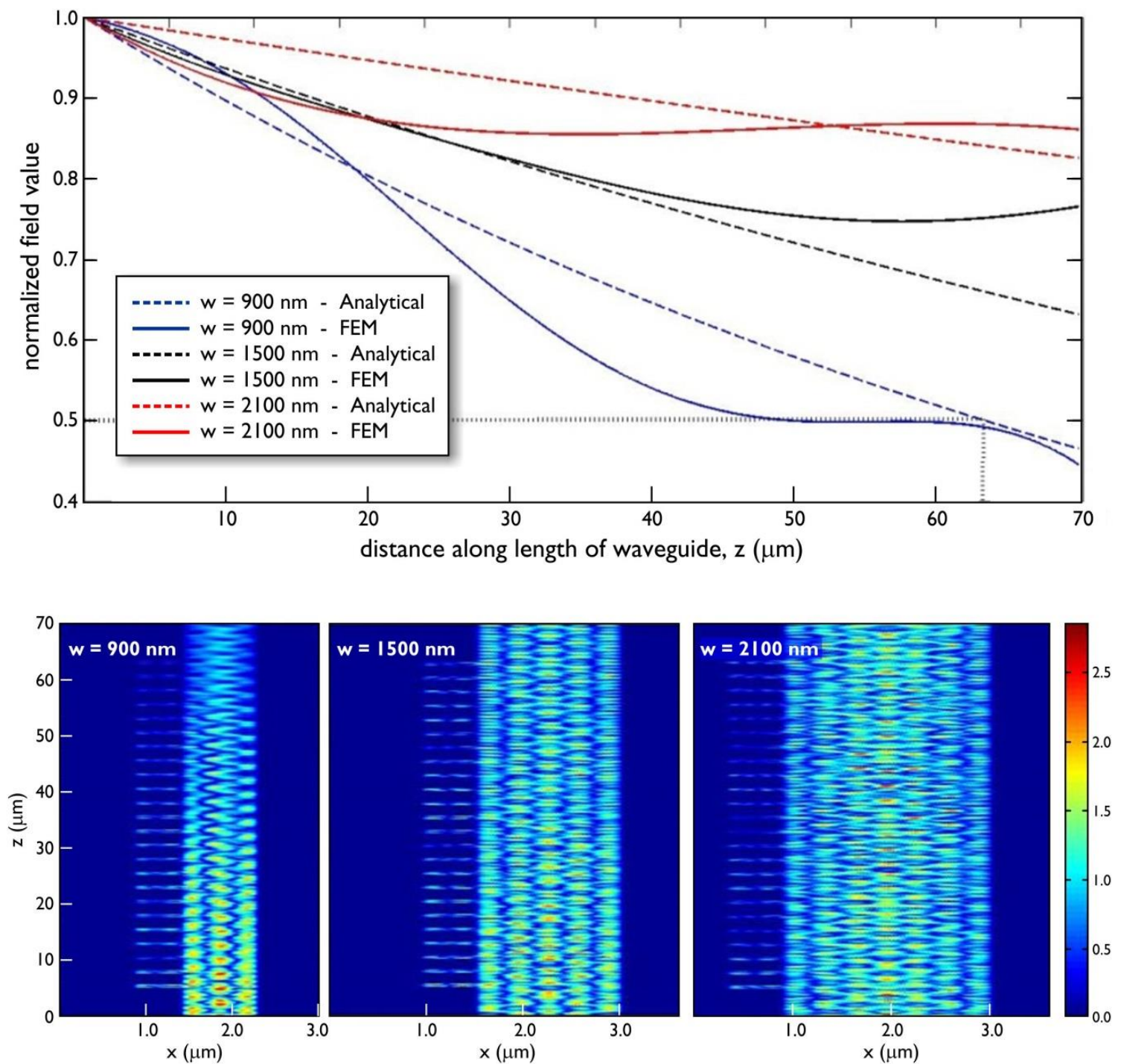

FIG. 9 a) The mean electric field magnitude along the length of the waveguide with 24 ZMWs, each separated by $\Lambda=2.5 \mu \mathrm{m}$. FEM calculations were used to find the fields for three different heights $(w)$ of the waveguide (solid lines) and it was also calculated analytically using Eq. (10) (dashed lines). The metal used was aluminum and the wavelength of light was $450 \mathrm{~nm}$. (b) FEM calculations with $d=900 \mathrm{~nm}, 1500 \mathrm{~nm}$ and $2100 \mathrm{~nm}$. A grating of period ( $p$ ) $600 \mathrm{~nm}$ was used to have a single mode ( $m=3,5$, 7 respectively). The dimensions of the bridge are $545 \times 545 \mathrm{~nm}^{2}$ and the wavelength of light is $450 \mathrm{~nm}$. 


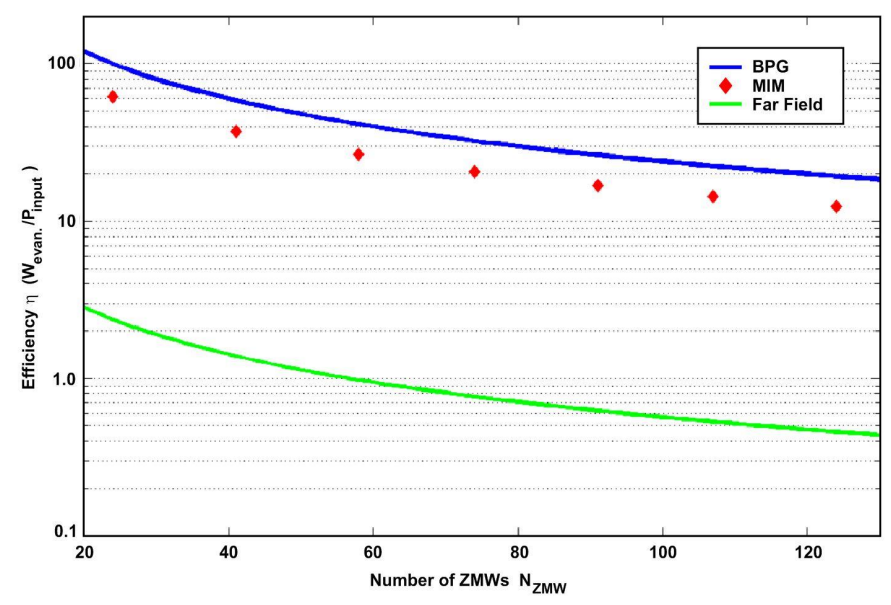

FIG. 10 The efficiencies of the MIM and BPG design compared to far field excitation. The values of the efficiencies for the grating design are calculated from Eq. (10). The efficiencies for the MIM design are also calculated from Eq. (10) with the values of $w$ corresponding to each $N_{Z M W}$ from Table 1 . The values are for the design wavelength of $\lambda=450 \mathrm{~nm}$.

The energy density inside a single ZMW is then given by

$$
W_{\text {evan }}=I_{\text {evan }} / c=\frac{P_{\mathrm{ZMW}}}{A_{\mathrm{ZMW}} \cdot c}=\frac{\eta_{\text {Airy }}}{A_{\mathrm{ZMW}} \cdot c} \frac{P_{\text {input }}}{N_{\mathrm{ZMW}}}
$$

resulting in an efficiency for far field (FF) illumination of:

$$
\eta_{\mathrm{FF}} \equiv \frac{W_{\text {evan }}}{P_{\text {input }}}=\frac{\eta_{\text {Airy }}}{c \cdot A_{\mathrm{ZMW}} \cdot N_{\mathrm{ZMW}}}=\frac{56.7}{N_{\mathrm{ZMW}}}\left[\frac{\mathrm{J} / \mathrm{m}^{3}}{\mathrm{~W}}\right]
$$

These excitation efficiencies are plotted in Figure 10. From the figure it is immediately evident that the excitation of a ZMW using the BPG or MIM design is more than an order of magnitude more efficient as compared to far field illumination. A typical fluorophore saturation intensity equals $1 \mathrm{~mW} / \mu \mathrm{m}^{2}$, requiring an evanescent energy density of approximately $3.3 \mathrm{~J} / \mathrm{m}^{3}$. In order to have $100 \mathrm{ZMWs}$ excited with this energy density, according to Figure 10, we need an overall laser input power of $0.14 \mathrm{~W}, 0.25 \mathrm{~W}$ and $6.2 \mathrm{~W}$ respectively for the BPG, MIM waveguide design and far field illumination.

Although both the BPG and the MIM waveguide approaches result in an increase of excitation efficiency of more than a factor of 10 , the underlying physical mechanisms are quite different. In the BPG the efficiency increase is mainly governed by the reduction of the "focused" spot size just above the ZMWs. This is due to the abundance of high diffraction angles close to 90 degrees inside a high refractive index substrate, and being equivalent to a $N A>1$ focusing. In the MIM waveguide approach the increase in excitation efficiency is based upon "recycling" of the input beam. All the energy contained in the input beam is coupled into the MIM waveguide mode that subsequently couples to all the bridge waveguides and ZMWs. The reason that the efficiency for MIM waveguide excitation is not higher and decreases with the number of ZMWs is mainly due to the absorption of the energy by the waveguide metal.

For practical purposes the proposed structures can be fabricated with modern fabrication techniques, as we took manufacturing tolerances into account when choosing actual design parameters. The BPG structure consists of a simple grating placed on a substrate of width around $33 \mu \mathrm{m}$ which is straightforward to fabricate and the efficiencies as calculated should be realized in a practical situation. For the MIM waveguide, the only stringent criterion for reaching a high efficiency is the presence of a single propagating mode in the waveguide. In practical situations, this can be achieved by the use of a grating at the input of the waveguide, as proposed here. Actual verification and fine-tuning of the simulations will be done in a future experimental study.

\section{CONCLUSIONS}

In this paper we studied the excitation efficiency of zero mode waveguides, using two different types of diffractive structures. Both the binary phase grating (BPG) and the metalinsulator-metal (MIM) waveguide concepts are based on the fact that light inside the evanescent field of a ZMW is not propagating and, by proper optical design, the ZMW input power can be reused for excitation of other ZMWs. The binary phase grating uses multiple reflections between the grating and the metal interface of the ZMW structures, whereas the MIM design relies on mode propagation inside a metallic waveguide. Using analytical reasoning and with the help of FEM simulations we showed that the excitation efficiency of zero modes waveguides can be increased with a factor of 20 or more compared to plain far field focusing onto the ZMW array. These zero mode waveguide arrays are being used in e.g. DNA sequencing. From an application point of view, this results in at least a factor of 10 increase in measurement throughput or a considerable cost reduction by the use of less powerful laser systems. Such methods of excitation of ZMWs can open up new avenues for further research. E.g. 2D ZMW structures could be used instead of the slit approach that is currently studied. First preliminary 2D calculations using smaller grid dimensions (in order to circumvent memory constraints) indeed suggest that a further increase of the throughput is to be expected using 2D diffracting structures.

\section{ACKNOWLEDGEMENTS}

The authors would like to thank dr. Gert 't Hooft for valuable discussions and careful reading of the manuscript.

\section{A BACK-SCATTERING FOR THE BRIDGE-WAVEGUIDE STRUCTURE}

If there are no dielectric perturbations in a waveguide, the modes of the waveguide are orthogonal and independent of each other. However any change from the ideal (unperturbed) waveguide will perturb the modes and couple energy between them. Using mode orthogonallity and considering that the perturbation causes slow variations in the amplitude we can derive the equation for changes in the amplitudes of the forward $\left(A^{+}\right)$and the backward $\left(A^{-}\right)$going waves as [16]:

$$
\begin{aligned}
& \frac{\partial A^{-}}{\partial z} \exp j(\beta z+\omega t)-\frac{\partial A^{+}}{\partial z} \exp -j(\beta z-\omega t)= \\
& -\frac{j}{2 \omega} \frac{\partial^{2}}{\partial t^{2}} \int_{\infty} P_{\text {pert }}(x) \cdot E_{y, m_{p}}(x) d x
\end{aligned}
$$


where $P_{\text {pert }}$ is the polarization perturbation given by the product of the change in the dielectric constant $\Delta \epsilon=\epsilon_{0} \cdot\left(n_{\text {dielectric }}^{2}-n_{\text {metal }}^{2}\right)$ and the forward going wave of the unperturbed waveguide, $A^{+} E_{y, m_{u}}(x, z, t)=A^{+} E_{y, m_{u}}(x) \exp j(-\beta z+\omega t)$. Here, $E_{y, m_{p}}(x)$ is the electric field of the perturbed waveguide, $n_{\text {dielectric }}$ and $n_{\text {metal }}$ are the refractive indices of the metal and the dielectric core of the waveguide respectively. Note that $\Delta \epsilon$ is zero everywhere else apart from the perturbed region $(-a / 2<x<a / 2 ;-w / 2<z<w / 2+d)$ and $E_{y, m_{u}}(x)$ is zero everywhere else apart from the unperturbed waveguide $(-w / 2<z<w / 2)$. The modes of the system are denoted by the index $m$ with subscripts $u$ and $p$ representing the unperturbed and perturbed waveguides respectively.

We can see (Figure 11) that the unperturbed waveguide can support only a single mode, corresponding to the thickness $w$ which was excited using the grating as described. Due to the perturbation, modes corresponding to thickness $w+d$ are also introduced to the waveguide and the coupling of these new modes to the mode of the unperturbed waveguide will result in the backscattering.

We assume that the forward wave does not change in amplitude during propagation and so $\partial A^{+} / \partial z=0$. The field distribution for the perturbed and the unperturbed waveguide is given by Eq. (5) and can be written as

$$
\begin{gathered}
E_{y, m_{\Sigma}(x)}=a_{m_{\Sigma}} u_{m_{\Sigma}}(x) \\
u_{m_{\Sigma}(x)}= \begin{cases}\sqrt{\frac{2}{d_{\Sigma}} \cos \left(\frac{m_{\Sigma} \pi x}{d_{\Sigma}}\right)}, & m_{\Sigma}=1,3,5 \ldots \\
\sqrt{\frac{2}{d_{\Sigma}} \sin \left(\frac{m_{\Sigma} \pi x}{d_{\Sigma}}\right)}, & m_{\Sigma}=2,4,6 \ldots\end{cases}
\end{gathered}
$$

where $m_{\Sigma}=m_{p}$ for the modes of the perturbed waveguide and $m_{\Sigma}=m_{u}$ for the single mode of the unperturbed waveguide and $d_{\Sigma}=d$ for the unperturbed waveguide and $d_{\Sigma}=d+w$ for the perturbed waveguide. Using Eq. (12) we get

$$
\begin{aligned}
A^{-}= & \frac{-j \omega \Delta \epsilon A^{+}}{2} \\
& \times \int_{-a / 2}^{a / 2} \exp (j 2 \beta z) d z \int_{-d / 2}^{d / 2} E_{y, m_{u}}(x) E_{y, m_{p}}(x) d x
\end{aligned}
$$

Considering we have an odd mode with propagation constant $\beta$ propagating in the unperturbed waveguide we have

$$
A^{-}=\frac{-j \omega \Delta \epsilon A^{+}}{2} \sin \beta a \int_{-d / 2}^{d / 2} \cos \left(\frac{m_{u} \pi x}{d}\right) E_{y, m_{p}}(x) d x
$$

Using Eq. (14) for $E_{y, m_{p}}(x)$ we get for $m_{p}=1,3,5 \ldots$

$$
\begin{gathered}
A^{-}=\text {Constant } \times \sin (\beta a)\left\{\frac{\sin \left(\frac{C_{1} d}{2}\right)}{C_{1}}+\frac{\sin \left(\frac{C_{2} d}{2}\right)}{C_{2}}\right\} \\
C_{1}=\frac{m_{p} \pi}{d+w}-\frac{m_{u} \pi}{d} ; \quad C_{2}=\frac{m_{p} \pi}{d+w}+\frac{m_{u} \pi}{d}
\end{gathered}
$$
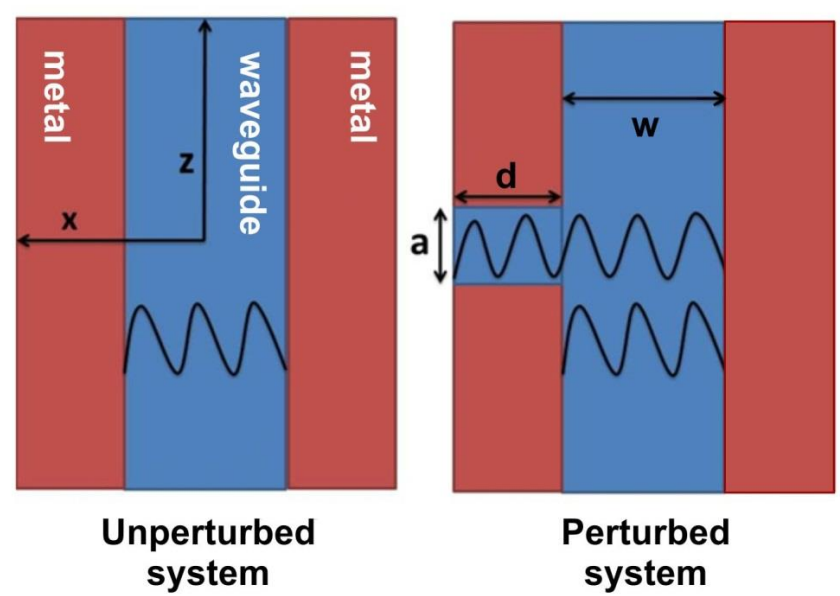

FIC. 11 The schematic of the unperturbed waveguide and the perturbed waveguide with the bridge. Only one mode is allowed to propagate in the unperturbed waveguide. The perturbed region can be considered as a waveguide with thickness $d+w$ and thus introduces new modes into the waveguide.

\section{References}

[1] M. Margulies. M. Egholm, W. E. Altman, S. Attiya, J. S. Bader, L. A. Bemben, J. Berka, et al., "Cenome sequencing in microfabricated high-density picolitre reactors," Nature 437, 376-380 (2005).

[2] D. R. Bentley, S. Balasubramanian, H. P. Swerdlow, G. P. Smith, J. Milton, C. G. Brown, K. P. Hall, et al., "Accurate whole human genome sequencing using reversible terminator chemistry," Nature 456, 53-59 (2008).

[3] K. J. McKernan, H. E. Peckham, G. L. Costa, S. F. McLaughlin, Y. Fu, E. F. Tsung, C. R. Clouser, et al., "Sequence and structural variation in a human genome uncovered by short-read, massively parallel ligation sequencing using two-base encoding," Genome Res. 19, 1527-1541 (2009).

[4] D. Lipson, T. Raz, A. Kieu, D. R. Jones, E. Giladi, E. Thayer, J. F. Thompson, et al., "Quantification of the yeast transcriptome by single-molecule sequencing," Nat. Biotechnol. 27, 652-658 (2009).

[5] J. Eid, A. Fehr, J. Gray, K. Luong, J. Lyle, G. Otto, P. Peluso, D. Rank, et al., "Real-time DNA sequencing from single polymerase molecules," Science 323, 133-138 (2009).

[6] M. J. Levene, J. Korlach, S. W. Turner, M. Foquet, H. G. Craigshead, and W. W. Webb, "Zero-mode waveguides for single-molecule analysis at high concentrations," Science 299, 682-686 (2003).

[7] P. M. Lundquist, C. F. Zhong, P. Zhao, A. B. Tomaney, P. S. Peluso, J. Dixon, B. Bettman, et al., "Parallel confocal detection of single molecules in real time," Opt. Lett. 33, 1026-1028 (2008).

[8] B. A. Flusberg, D. R. Webster, J. H. Lee, K. J. Travers, E. C. Olivares, T. A. Clark, J. Korlach, and S. W. Turner, "Direct detection of DNA methylation during single-molecule, real-time sequencing," Nat. Methods 7, 461-465 (2010).

[9] R. G. Blazej, P. Kumaresan, and R. A. Mathies, “Microfabricated bioprocessor for integrated nanoliter-scale Sanger DNA sequencing," Proc. Nat. Acad. Sciences 103, 7240-7245 (2006).

[10] E. Servoli, H. Feitsma, B. Kaptheijns, P. J. van der Zaag, and R. Wimberger-Friedl, "Improving DNA capture on microarrays by integrated repeated denaturing," Lab Chip. 12, 4992-4999 (2012).

[11] M. Xu, H. P. Urbach, D. K. G. de Boer, and H. J. Cornelissen, 
"Wire-grid diffraction gratings used as polarizing beam splitter for visible light and applied in liquid crystal on silicon," Opt. Express $13,2303-2320$ (2005).

[12] M. G. Moharam, E. B. Grann, D. A. Pommet, and T. K. Gaylord, "Formulation for stable and efficient implementation of the rigorous coupled-wave analysis of binary gratings," J. Opt. Soc. Am. A 12, 1068-1076 (1995).

[13] X. Wei, A. J. H. Wachters, and H. P. Urbach, "Finite-element model for three-dimensional optical scattering problems," J. Opt. Soc. Am. A 24, 866-881 (2007).
[14] A. Yariv, and P. Yeh, Optical Waves in Crystals (Wiley Interscience Publication, Hoboken, 1984).

[15] I. P. Kaminow, W. L. Mammel, and H. P. Weber, "Metal-clad optical waveguides: analytical and experimental study," Appl. Optics 13, 396-405 (1974).

[16] C. Pollock, and M. Lipson, Integrated Photonics (Kluwer Academic Publishers, Dordrecht, 2003). 